\title{
Application and Research of Geologic Radar in Groundwater Disease of Tunnel Engineering Inspection
}

\author{
WANG Yu ${ }^{1,2}$, WU chuang-sheng ${ }^{2}$, Shu Zongying ${ }^{1 *}$ \\ ${ }^{1}$ Shanghai Urban Construction Vocational College, 200438, Shanghai, china \\ ${ }^{2}$ Chongqing Jiaotong University, 400074, Chongqing, china
}

\begin{abstract}
As a kind of underground lineament building, the tunnel inevitably will pass through different hydrology geology in construction process and thus form a catchment corridor which usually occurs in most of tunnels. Pour water and waterlogging is a common geological disaster and the main disease both in the construction tunnels and operation tunnels. So it is urgent for us to carry through the research on the geological disaster of groundwater. The Inspecting and controlling measures against the ground water geological disaster are also needed. In this paper, Geologic Radar is applied to tunnel engineering quality inspection to detect some quality problems possibly caused by groundwater. In order to diagnose the disease degree of operation tunnels and to estimate tunnel's security condition, this paper analyzes the causes of bugs existing behind the tunnel lining, expounds the theory and method of making non-destructive test on tunnel lining quality by using Geological Radar. By processing and analyzing field data, the following functions can be performed, such as detecting the thickness of concrete lining accurately, determining the distribution position and quantities of rebar and grid steel, searching existed cavity and uncompacted area behind the lining especially lining arch top. We also apply the method of morphology to the extraction of liner image feature. We get the liner boundary line and waterlogging area more intuitively by wavelet analysis, and find the anomalous point in the liner. It has been proved that Geologic Radar is a feasible method to inspect the structure of tunnel concrete lining. Adopting hole-drilling method to make verifications with detecting results of geological radar, the results of the two kinds of methods do not have big difference.
\end{abstract}

\section{GEOLOGIC RADAR DETECTION METHOD AND WAVEFORM RECOGNITION}

\subsection{Detection Principle and Function}

Geologic Radar uses transmitting antenna that emits high frequency electromagnetic wave, which is in a broadband pulses form and in the buried targets direction. The electromagnetic wave will create reflection, when passing the discontinuity of dielectric, and be received by receiving antenna. Through the analysis of the transmit character of electromagnetic wave in time and space, the information about the spatial location,scale, physical properties etc., of the buried targets will be obtained. Electromagnetic wave propagating in the medium, its path and electromagnetic wave strength varied with electrical properties and the geometry. The detection resolution depends primarily on pulse width and dielectric absorption characteristics; probing depth closely related to the center frequency .The detection principle shown in Figure $1^{[1][2] .}$
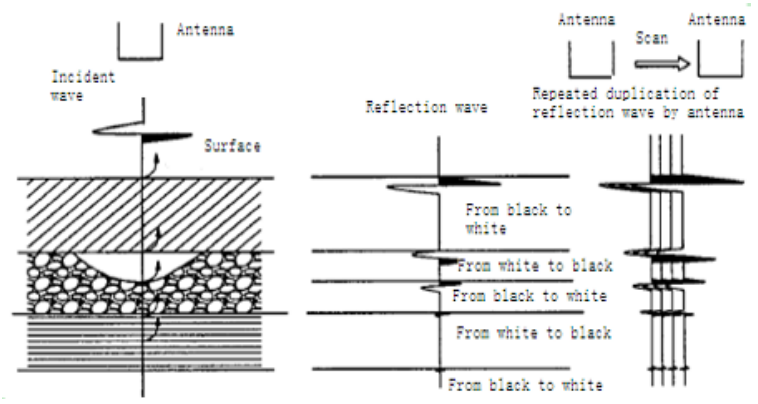

Fig.l Simplified schematic of ground probing radar

The common problems of tunnel concrete lining quality is: lining behind the back fill is not dense enough lining thickness, leakage of water, local cracks and inadequate reinforcement layout and so on. using ground penetrating radar technology, you can achieve the following objectives:

1) The detection of empty existence behind tunnel lining and the location and scope of backfilling nondense point

2)The detection of concrete lining and the secondary lining thickness;

3) The detection of reinforced concrete lining and distribution of steel grating. 
4) The detection of cracks in concrete secondary lining.

5) The detection of interlayer water ${ }^{[3]}$

How to detect the initial tunnel lining and the stagnant water surrounding rock hole accurately is key to the proper application of treatment methods for tunnel leak, while also ensuring the effect of leakage control in a certain extent, which make treatment project more economical and effective. Water lag behind the reinforced concrete layer, coupled with empty form, and the irregular nature of water inside the cave, may be filled with air, slurry, soil factors, such as media, so there is considerable complexity.

\subsection{A typical target identification and to determine the waveform}

Lining thickness detection: There is a big difference in composition and physical properties and dielectric constant between the lining and rock, especially between the beginning of lining and the rock. Waves from the concrete into the surrounding rock, the reflected wave amplitude significantly increased, the video rate receded. The thickness of the layer of concrete tunnel structure is based on the electromagnetic pulse in the structure of the interface layer of the reflection time and various structural layers in the propagation velocity of electromagnetic waves calculations. The key to determine the thickness is the calculation of electromagnetic waves travel time in the tunnel's structural layer. Then according to the electromagnetic propagation velocity in concrete $(v=0.12 \mathrm{~m} / \mathrm{ns})$ to calculate the thickness of structural layers.

The empty area detection: Dielectric constant is difference between the air and lining and rock, when the tunnel lining and surrounding area have vacant area, due to differences in different media, the result of electromagnetic geological Radar reflectivity waveform distortion is more evident, reflecting the positive and negative waves is interval, highly visible, easily identified, when vacant area exist in the lining and the rock, reflected wave will show a complex vibration effects, interior reflected waves increase, the cavity waveform was hyperbolic shape.

Detection of water-rich region: The wave impedance differences are also large between water and concrete and rock, positive and negative reflected wave is interval, and gray and white is interval by wave-phase and reflecting very strong. Velocity and attenuation of radar reflection wave is significantly faster in dry rock in the of water-rich rock.

\section{2 image enhancement processing based on wavelet technology}

Wavelet transform have multi-scale analysis and good energy compactness ${ }^{[4,5,6]}$, that is, Wavelet have the capability of time-frequency localization and multiresolution analysis. In the tunnel empty and water detection, using wavelet transform image processing, can both improving the image visual effect and the clarity of the image elements and make images become more conducive to the computer's processing analysis. For example: for the geological radar images of lining, consider lining the boundary with the surrounding rock, we need the integrity of strong lining to distinguish between lining and surrounding rock; when we need to consider the defects inside lining and detected the noncompact of the zone, integrity is not that we need to consider, but is concerned about the structure of the internal detail. In this paper, wavelet transform will be used to image processing, such as the detail on the lining inside the structure, non-dense areas, reinforcing bars.

The usual processing techniques can be divided into image-based domain and transform domain. The first method is directly operations by pixel point, the latter is a more complex approach. The latter method firstly transform image from space domain to another domain representation, by amending corresponding coefficient to increase the output image purposes. The latter method applied here for the geological radar image processing.

How to use Wavelet-based multi-scale method for image enhancement? Firstly, we use wavelet transform technology to decomposed of image with the different size, location and orientation, and then change some of the weight coefficient with processing requirements, enhancing the interesting part, weakening other parts; Wavelet method can be decomposed into different frequency signals for variety of different frequency composition of mixed-signal, and thus can be effectively applied to the letter-noise separation, edge detection and image restoration. Here we put the brightness of the original image as $\mathrm{B}(\mathrm{x}, \mathrm{p})$, wavelet function as $\mathrm{f}(\mathrm{x}, \rho)$, then enhancing brightness $\mathrm{B} /(\mathrm{x}, \rho)$ can be derived from the first two convolution:

$$
B^{\prime}(\bar{r})=f(x, \bar{r}) \times B(x, \bar{r})
$$

Here we used is the db series "db5", the general wavelet decomposition with the following diagram to represent.

Table 1. Breakdown Structure of Two-Layered Wavelet

\begin{tabular}{|l|l|l|}
\hline$D_{J-1}^{H L}$ & $D_{J-1}^{H H}$ & \multirow{2}{*}{$D_{J}^{L H}$} \\
\cline { 1 - 2 }$C_{J-1}$ & $D_{J-1}^{I H}$ & \\
\cline { 1 - 1 } & \\
\hline \multicolumn{2}{|c|}{$D_{J}^{H L}$} & $D_{J}^{H H}$ \\
\hline
\end{tabular}

DWT (wavelet transform), an image is decomposed into the size, location and orientation in different scales, so that can selectively amplify the quantity of interest and reduce unwanted weight. The image noise will also have high-frequency information, and enhanced process may amplify noise. Space-frequency analysis method could enhancing the image edge, at the same time, we need to consider the noise issue, so the use of images should be the wavelet adaptive treated image. When Use ground penetrating radar to processing image, we should 
increase and indicate pixel point, so that these abnormal points and steel location can be find,. Put the image "db5" two-level decomposition, select the appropriate range of values of pixels after transformation, and the enhancement factor for strengthening. Pixel treatment effects showed in Figure 2 and Figure 3.

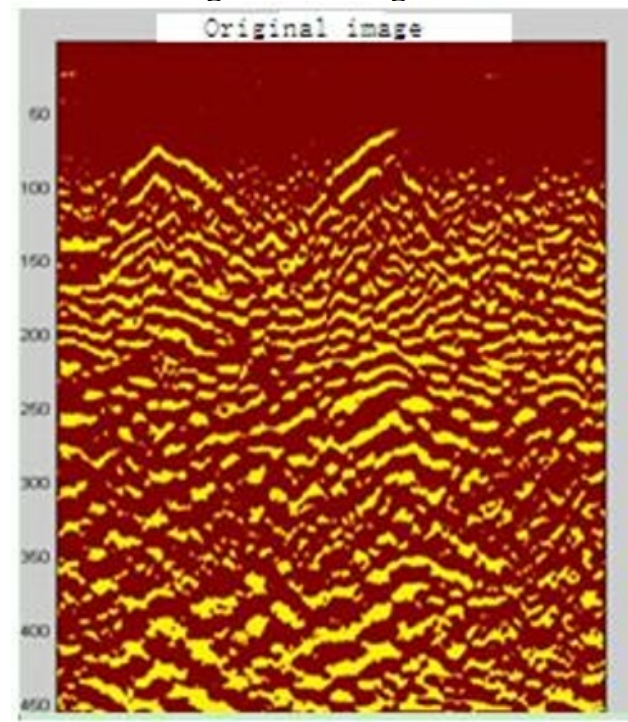

Fig.2 disposal map of Pixel Dot

By contrast, we can see that the number of abnormal body increased significantly in enhanced graph, and it could help us to observe loose area of lining. The detection of unusual data of lining one by one can make us to find the corresponding pixel values of the characteristics

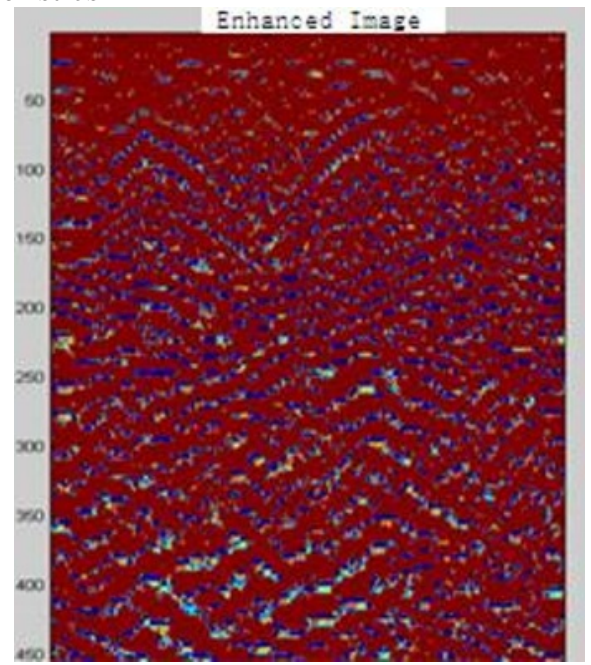

Fig3 disposal map of Pixel Dot

\section{Examples of engineering application}

\subsection{Project Overview}

In Guizhou Province, a tunnel is 360 meters long, the original design is masonry arch. As the tunnel collapsed imports, the import segment added a 50-meter reinforced concrete, and construct addition of $10 \mathrm{~cm}$ masonry to reinforced concrete waterproofing layer. The tunnel is not established drainage measures on both sides, because of the recent rains, poor drainage, resulting in side-wall of water seepage, road arch crack.

\subsection{Detection Program}

Adopt of the U.S. SIR-2000 model ground penetrating radar to detect the tunnel. Because the Longer operating life of the tunnel, and the related design documents missing, with the actual situation, we formulate the following test program: Make three waist line scan for the vault, and both sides of the arch of the tunnel, mainly detected disease such as lining arch ring thickness, steel distribution and empty area. Select the $900 \mathrm{MHz}$; scanning both sides of a two-line, scanning the two roads to two-line, and scanning the rock and road test under the water-rich situations, which needs a larger detection depth, selecting the $400 \mathrm{MHz}$ antenna.

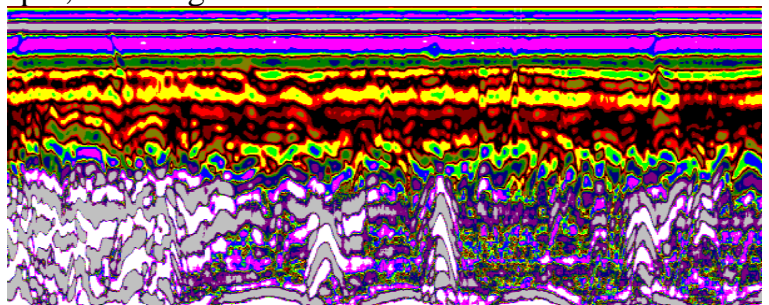

Fig.4 ground penetrating radar scanning map of pavement in tunnel

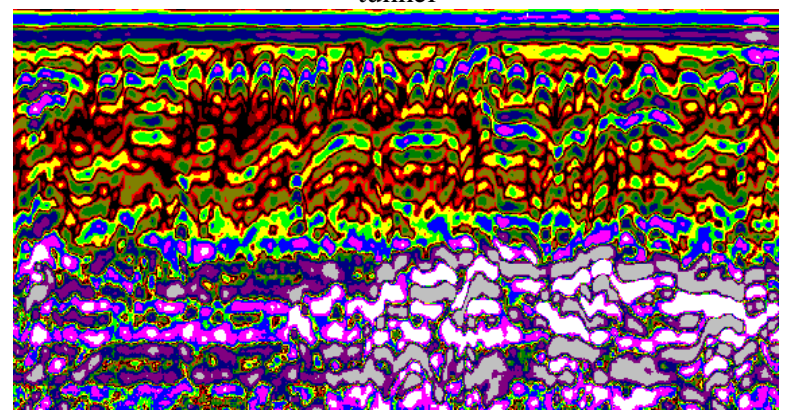

Fig.5 ground penetrating radar scanning map of tunnel vault

\subsection{Test results}

Analyzing the record data by the radar waveform recognition methods and using wavelet technology processing to image enhancement, test results obtained are as follows: Main Arch Tunnel Entrance Section 0-50 meters is reinforced concrete, the average thickness of $50 \mathrm{~cm}$, the average distance between reinforcement 30 $\mathrm{cm}$, there is a slight segregation of reinforced concrete, and have not found more serious deficiencies. In $\mathrm{k} 50 \mathrm{~m}-\mathrm{k} 340 \mathrm{~m}$, section of masonry arch vault and two waist average thickness was $35 \mathrm{~cm}$, reinforced concrete and waterproofing layer average thickness was $15 \mathrm{~cm}$, steel spacing is $40-45 \mathrm{~cm}$ in waterproof layer, vault and two lumbar have severe empty walls; There was a row of empty areas distribution behind tunnel lining, the empty in vault and in arch of lumbar total length was $35 \mathrm{~m}$, accounting for $12 \%$. The largest hole body was in uplink K105-K113m, and in downlink K45-K60m, with a total extension of $15 \mathrm{~m}$, the rest of the lot behind the lining was fundamental compact, less adverse conditions. In order to verify test results of ground penetrating radar, we drill holes during $45-60 \mathrm{~m}$. and 
found $38 \mathrm{~cm}, 42 \mathrm{~cm}$ deep of the hole. Test results showed that: the tunnel side walls on both sides of the average thickness was $50 \mathrm{~cm}$, reinforced concrete and waterproofing layer average thickness was $25 \mathrm{~cm}$, There is the phenomenon of stagnant water both in back wall and side walls ; surface layer (due to road damage, it conducted two paved) of The average thickness was $75 \mathrm{~cm}$, The stagnant water exist below the road which show in Figure 4 and Figure 5. Treatment was validated the test results, in the back leakage regulation of the lining, the engineering control methods are: chemical grouting; filleting plugging; chisel Comprehensive Treatment of the drainage trough.

\section{$4 \quad$ ONCLUSIONS RECOMMENDATIONS}

Geological radar is based on difference electromagnetic of the body and its surrounding rocks the electrical characteristics, buried depth, size and other characteristics of size and speciation methods have a direct impact on the effectiveness of Geological radar, while, due to the complexity of geological structure, liking other geophysical methods, geological radar is limitations, multi-solution, one-sidedness, Only by constantly improving the performance of the instrument itself, and combining of the known geological data, and reasonably deducing from geological radar data, it could play better role of detection .Thus, in practice, we should focus more on site-specific geophysical characteristics, through on-site tests to correctly select the operating parameters of geological radar in order to avoid an unreasonable or wrong choice of technical parameters which lead to application failure.

In the tunnel project, if the lining construct very perfect, the second lining interface is not very easy to see (first and second lining is the same medium, there will be no significant reflection layer). However, in accordance with its shortcomings (layer hollow, off air, non-dense, etc.) to find the reflection interface. If the tunnel surface is uneven, especially on the ground is not flat, it will causing great difficulties to the radar detection. This time should observe the working conditions of the antenna (whether to leave the tunnel surface, it suddenly moved to another place, etc.) and note the abnormal Stake of the antenna in order to make the right judgments.

For the lining of seepage, leakage, leakage as well as the lining of the plot behind the empty water diseases, some cracks will also contain water inside the cave, other than the media, and form into diverse, with varying degrees of water. In addition, outside of random interference, for example, the radar may be quite sensitive because of the steel lining the track network and the outside metal. Interpretation of existing data should have a general understanding, coupled with adequate pit exploration drilling or work, in order to enter the stage of quantitative interpretation. To what is known as the basis for geological interpretation; geological interpretation of the results can be used to verify by drilling hole, drilling results and then fed back into the geological interpretation, and therefore the geological interpretation has always been a constantly updated to improve the process.

\section{Acknowledgement}

Project supported by Key Laboratory of Geohazard Prevention and Geoenvironment Protection (No. gsgzj2016-01)

\section{References}

1. Li da-xin. Ground Penetrating Radar Methods and Applications [M]. Geological press, 1994.

2. WU Jung, MAO Hai-he, YING Song, Application of ground probing radar to short-term geological forecast for tunnel construction[J] Rock and Soil Mechanics, 2003,s(1): 154-157.

3. LI Jin-ping, SHAO Pi-yan, GU Mu, Application and Analysis of GPR in Railway Tunnel Engineering Quality Inspection[J] CHINA RAILWAY SCIENCE2006,27(2),56-59.

4. S.Ma11at.A theory for multiresolutiun signal decomposition: the wavelet representation. IEEE Trans, PAMI, 1989.11(7):673-696.

5. S.Mallat.. Characterization of signals from multiscale edges. IFEE Tans,PAMI,1992,t4(7):711-733.

6. I.Daubechies. Ten Lectures on Wavelets. Philadeiphia. PA:SIAM, 1992: 143-158.

7. Donoho DL.De-Noising by Soft-Thresholding. IEEE Trans, on Inform,Theory,1994: 123-142. 